\title{
Head Math Model For The Low-Flow Impellers Of The Centrifugal Compressors
}

\author{
Vyacheslav Ivanov ${ }^{1,}$ Yuri Kozhukhov ${ }^{1, *}$, and Minh Hai Nguyen ${ }^{2}$ \\ ${ }^{1}$ Peter the Great St. Petersburg Polytechnic University, St. Petersburg, Russian Federation \\ ${ }^{2}$ Petrovietnam Exploration Production Corporation, Ho Chi Minh City, Viet Nam
}

\begin{abstract}
A new mathematical model has been developed to determine the coefficient of internal and theoretical head in the variant design of the low-flow rate centrifugal compressors impellers. A parametric study of the flow part of the impeller is carried out. In total 1620 impellers are numericaly simulated. As a result, a numerical database of gas dynamic and geometric parameters was developed. Due to an a priori analysis, the relations of parameters with the geometric shape of the flow part are determined. The mathematical model is developed using gas-dynamic parameters and relations determined from numerical database. Using centrifugal compressor stage digital twins, a generalizing relationship has been developed to determine the complex of friction and leakage losses. The reliability of the math model is validated by the comparison with experimental data and the results of numerical experiment in digital twins, which are not involved in the model. The application of the head math model is determined in the range of the conditional flow coefficient $0.006<\Phi \mathrm{d}<0.02$ and the theoretical head coefficient $0.60<\psi \mathrm{t}<0.72$.
\end{abstract}

\section{Introduction}

There are no simple engineering methods for calculating the theoretical head coefficient and the complex of friction and leakage loss for low-flow rate stages. Most of the developed dependencies [1, 2, 3] relate to medium and high flow-rate stages and cannot be applied in designing process of low-flow rate stages. The flow features in low-flow rate stages $[4,5,6]$ are caused by the influence of the viscosity. It does not allow the use of methods for calculating inviscid flow, what is also shown in the work [7]. Methods for calculating a viscous boundary layer are quite complicated and are common only in research organizations and therefore are not widely used in practical applications. Experimental research requires expensive equipment. Experimental studies of many parameters as in work [8] require expensive equipment and much time. Modern commercial packages for modelling the workflow using computational gas dynamics methods are currently limited due to the need to train specialized personnel and the financial costs of acquiring equipment and licenses. Moreover, a number of scientific publications [9, 10, 11, $12,13,14,15,16,17]$ show the efficiency of using computational methods in the design and study of centrifugal compressors low-flow rate stages. Computational methods are also used in the calculation of such an important phenomenon as surging in centrifugal compressors [18, 19]. Computational methods are widely used in modeling the working processes of turbomachines [20,21].
The purpose of the study is to develop an engineering method for determining the internal and theoretical Euler head coefficient for the preliminary design of the centrifugal compressor impeller of a low-flow rate stage booster compressor station for a thermal power plant.

To achieve the goal of the study, it is required to solve the following tasks:

To carry out modelling of impellers in a wide changes range in geometric and gas-dynamic parameters.

To analyse the influence of parameters and derive generalized dependencies to determine the theoretical head coefficient and the friction and leakage loss complex.

Earlier in [22], it was shown that in order to simulate the working process with obtaining a gas-dynamic characteristic, it is sufficient to use a simplified calculation model of the impeller (see fig. 1). The change in the characteristic consists only in a shift along the flow axis. This can be taken into account by the introduced correction. The reliability of the calculations is also shown in comparison with experimental data using the k- $\omega$ turbulence model [23].

For the statistical priori analysis and the generalizing dependencies derivation, 1620 centrifugal impellers models of the low-flow rate centrifugal compressors stages were designed and modelled according to the simplified calculation model for the following range of geometric and gas-dynamic parameters: calculated conditional flow coefficient $\Phi_{\mathrm{d}}=0.004 \div 0.020$; conditional Mach number $M_{u}=0.5 \div 0.8$; the blades height ratio at the inlet and outlet $b_{1} / b_{2}=0.5 \div 1.0$; relative hub diameter $\bar{D}_{h}=0.3 \div 0.5$; the number of

* Corresponding author: kozhukhov_yv@mail.ru 
blades $z=12 \div 20$; blade outlet angle $\beta_{\mathrm{bl} 2}=30^{\circ} \div 50^{\circ}$. Table 1 shows the variable impeller parameters used in the parametric study. The calculation results were processed by [24]. The design of the impeller is carried out on the design mode, under the condition of shockfree entry into the impeller blade system.

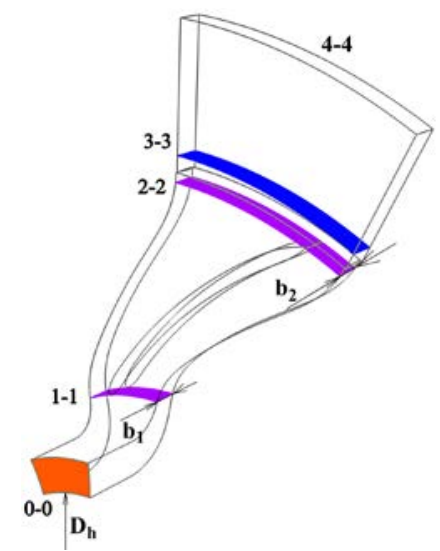

Fig. 1. Ansys CFX simplified three-dimensional computational domain of the impeller.

Table 1. Variable impeller parameters in a simplified numerical model.

\begin{tabular}{|c|c|c|c|c|c|c|}
\hline № & Parameter & $\begin{array}{c}\mathbf{1} \\
\text { step }\end{array}$ & $\begin{array}{c}\mathbf{2} \\
\text { step }\end{array}$ & $\begin{array}{c}\mathbf{3} \\
\text { step }\end{array}$ & $\begin{array}{c}\mathbf{4} \\
\text { step }\end{array}$ & $\begin{array}{c}\mathbf{5} \\
\text { step }\end{array}$ \\
\hline 1 & $\begin{array}{c}\text { Blade } \\
\text { number, z }\end{array}$ & 12 & 16 & 20 & - & - \\
\hline 2 & $\begin{array}{c}\text { Blade outlet } \\
\text { angle, } \beta_{\text {bl2 }}{ }^{\circ} \\
\text { (from } \\
\text { tangential } \\
\text { direction) }\end{array}$ & 30 & 40 & 50 & - & - \\
\hline 3 & $\begin{array}{c}\text { Relative } \\
\text { hub } \\
\text { diameter, } \overline{\mathrm{D}_{\mathrm{h}}}\end{array}$ & 0.30 & 0.40 & 0.50 & - & - \\
\hline 5 & $\begin{array}{c}\text { Ratio of } \\
\text { inlet to } \\
\text { outlet blade } \\
\text { height, b1/b2 }\end{array}$ & 0.50 & 0.75 & 1.0 & - & - \\
\hline 5 & $\begin{array}{c}\text { Conditional } \\
\text { Mach } \\
\text { Number, } \mathrm{M}_{\mathrm{u}}\end{array}$ & 0.50 & 0.60 & 0.70 & 0.80 & - \\
\hline 6 & $\begin{array}{c}\text { Conditional } \\
\text { flow rate } \\
\text { coefficient, } \\
\Phi_{\mathrm{d}}\end{array}$ & 0.004 & 0.008 & 0.012 & 0.016 & 0.020 \\
\hline
\end{tabular}

\section{Methods}

The geometric and gas-dynamic parameters were selected in the process of analysing.

Geometric diffusivity taking into account constraint is determined by the equation

$$
K_{\text {geom }}^{\prime}=\frac{\bar{D}_{1} \cdot \bar{b}_{1} \cdot \sin \beta_{\mathrm{bl} 1} \tau_{1}}{\bar{b}_{2} \cdot \sin \beta_{\mathrm{bl} 2} \tau_{2}} .
$$

The diffusivity factor characterizes the diffusivity of the interscapular channel at maximum velocity on the posterior surface of the impeller blade back side and is determined by the expression

$$
F_{D}=1-\frac{w_{2}}{w_{\max }}
$$

Flow diffusivity, as the ratio of relative velocities at the inlet and outlet of the impeller:

$$
n_{w}=w_{1} / w_{2} .
$$

As the analysis shows, the following parameters influence the amount of slope and the nature of the diffusivity factor dependence on geometric diffusivity (see Fig. 2, 4, 6, 8), taking into account constraint: the number of blades $z$, the outlet angle of the blades $\beta_{\mathrm{bl2}}$, the hub diameter $\bar{D}_{h}$ and the conditional Mach number $M_{\mathrm{u}}$. In this case, the dependence $F_{D}=f\left(K^{\prime}{ }_{g d}\right)$ remains linear and only the slope of the line and the value change.

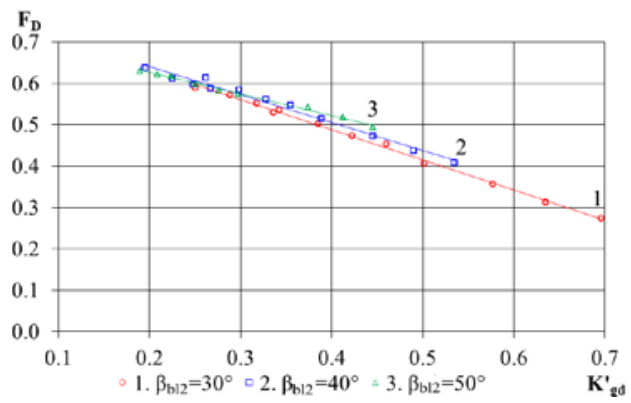

Fig. 2. Dependence of the diffusivity factor on the geometric diffusivity at the $\mathrm{z}=16, \overline{\mathrm{D}}_{\mathrm{h}}=0.4$ and variable $\beta_{\mathrm{bl2}}{ }^{\circ}$.

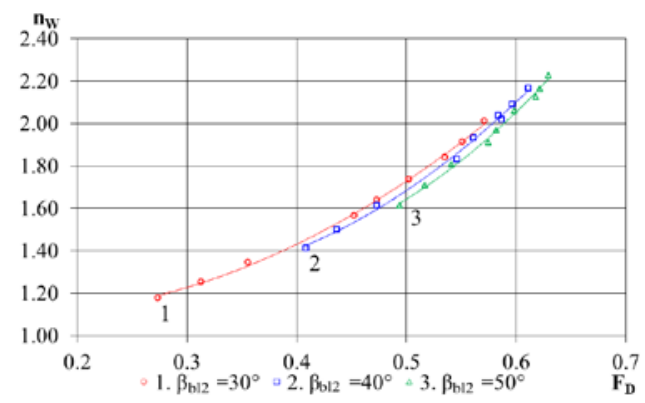

Fig. 3. Dependence of the flow diffusivity on the diffusivity factor at the $\mathrm{z}=16, \overline{\mathrm{D}}_{\mathrm{h}}=0.4$ and variable $\beta_{\mathrm{bl2}}{ }^{\circ}$.

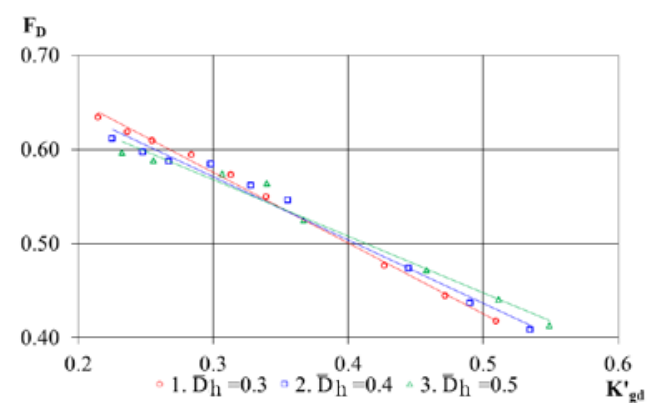

Fig. 4. Dependence of the diffusivity factor on the geometric diffusivity at the $\mathrm{z}=16, \beta_{\mathrm{bl} 2}=40^{\circ}$ and variable $\overline{\mathrm{D}}_{\mathrm{h}}$. 


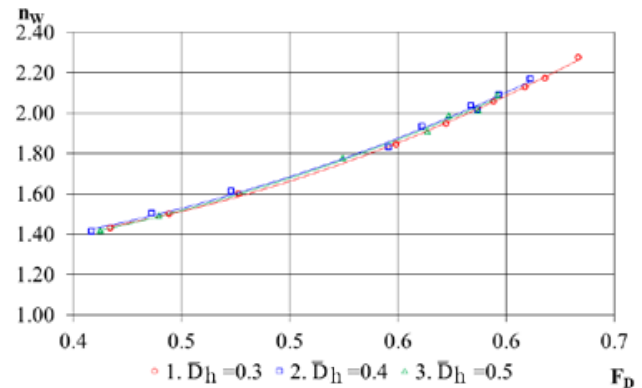

Fig. 5. Dependence of the flow diffusivity on the diffusivity factor at the $\mathrm{z}=16, \beta_{\mathrm{b} 2}=40^{\circ}$ and variable $\overline{\mathrm{D}}_{\mathrm{h}}$.

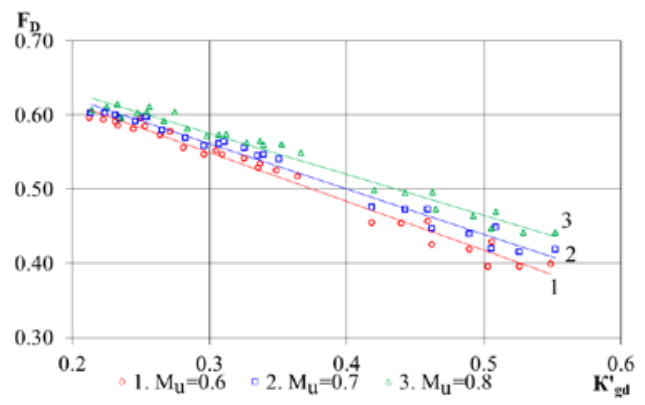

Fig. 6. Dependence of the diffusivity factor on the geometric diffusivity at the $\mathrm{z}=16, \beta_{\mathrm{bl} 2}=40^{\circ}$ and variable $\overline{\mathrm{D}}_{\mathrm{h}}$ and $\mathrm{M}_{\mathrm{u}}$.

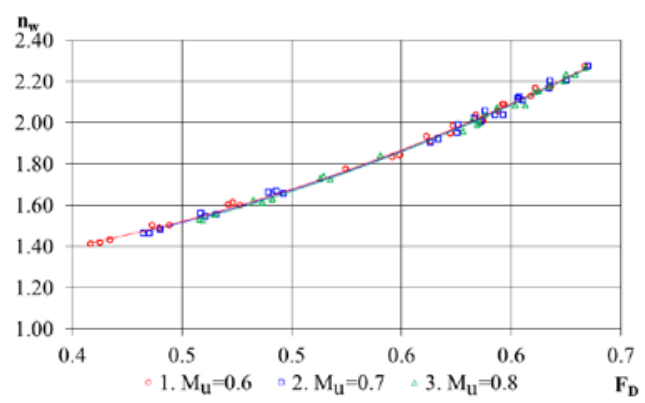

Fig. 7. Dependence of the flow diffusivity on the diffusivity factor at the $\mathrm{z}=16, \beta_{\mathrm{bl} 2}=40^{\circ}$ and variable $\overline{\mathrm{D}}_{\mathrm{h}}$ and $\mathrm{M}_{\mathrm{u}}$.

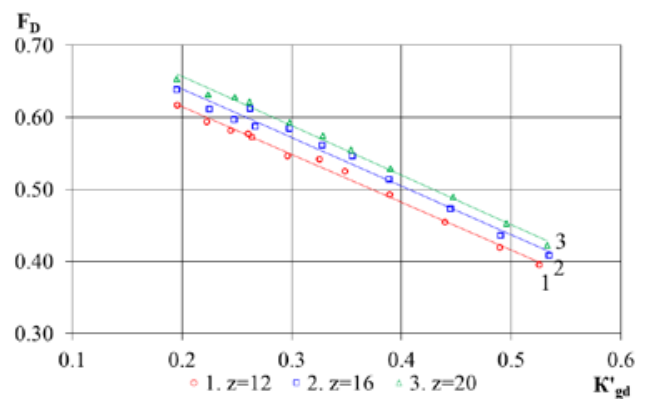

Fig. 8. Dependence of the diffusivity factor on the geometric diffusivity at the $\beta_{\mathrm{bl} 2}=40^{\circ}, \overline{\mathrm{D}}_{\mathrm{h}}=0.4$ and variable $\mathrm{z}$.

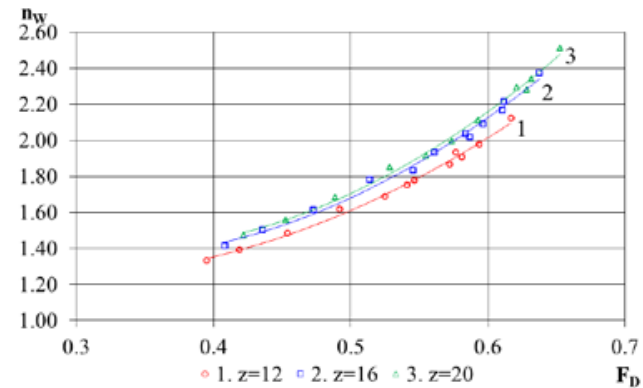

Fig. 9. Dependence of the flow diffusivity on the diffusivity factor at the $\beta \mathrm{bl2}=40^{\circ}, \overline{\mathrm{D}}_{\mathrm{h}}=0.4$ and variable $\mathrm{z}$.

A priori analysis found that there is a linear relationship between the diffusivity factor and the geometric diffusivity coefficient, taking into account constraint. Four parameters influence the magnitude and slope of the linear characteristic: the number of blades, the outlet angle of the blades, the hub diameter, and the conditional Mach number.

Expression for determining the diffusivity factor:

$$
\begin{aligned}
& F_{D}=\left(0,012 \cdot z+0,084 \cdot M_{u}+0,124 \cdot \beta_{b l 2 \text { (in rad.) }}+\right. \\
& \left.+0,084 \cdot \bar{D}_{h}-1,223\right) \cdot K_{g d}^{\prime}+0,77
\end{aligned}
$$

A nonlinear relationship in the form of a quadratic dependence is established between the flow diffusivity and the diffusivity factor. The influence of the relative hub diameter and the conditional Mach number can be neglected due to the small magnitude of the change in parameters. It is established that the number of blades and the outlet angle of the blades affect the change in the rate of change in flow diffusivity from the diffusivity factor. flow:

The expression for determining the diffusivity of the

$$
\begin{gathered}
n_{w}=\left(0,054 \times \beta_{\text {bl2 (in rad.) }}+0,381 \times \mathrm{z}+0,310\right) \times \mathrm{F}_{D}^{2}+ \\
+\left(0,318 \times \beta_{\text {bl2 (in rad.) }}-0,359 \times \mathrm{Z}+2,576\right) \times \mathrm{F}_{D}+ \\
+\left(-0,410 \times \beta_{\text {bl2 (in rad.) }}+0,096 \times \mathrm{z}+0,287\right)
\end{gathered}
$$

Having the value of the relative inlet velocity, it becomes possible to determine the coefficient of theoretical pressure by the equation:

$$
\psi_{\mathrm{t}}=1-\sqrt{w_{2}^{2}-\varphi_{2}^{2}} .
$$

The average relative error of theoretical pressure coefficient approximation by the presented method is $\delta$ $\left(\psi_{t}\right)=0.65 \%$. The value of the mean square dispersion is $\mathrm{R}^{2}=0.988$.

The results of model stages digital twins modeling of the Dresser\&Clark series $Q, R, S, T, U, V$ are used. Based on three-dimensional modeling of the workflow in detailed models of 18 low-flow rate stages, a relationship has been developed to determine the complex of friction and leakage loss:

$$
\beta_{\text {fr }}+\beta_{\text {leak }}=0,0118 \cdot\left(F_{2} / F_{\text {axial }} \cdot \varphi_{2} \cdot \psi_{t}\right)^{-0,9} \text {. }
$$


In (7) $F_{2} / F_{\text {axial }}$ is a ratio of the areas at the impeller outlet and the axial gaps inlet on the shroud and hub side, $\varphi_{2}$ - flow coefficient.

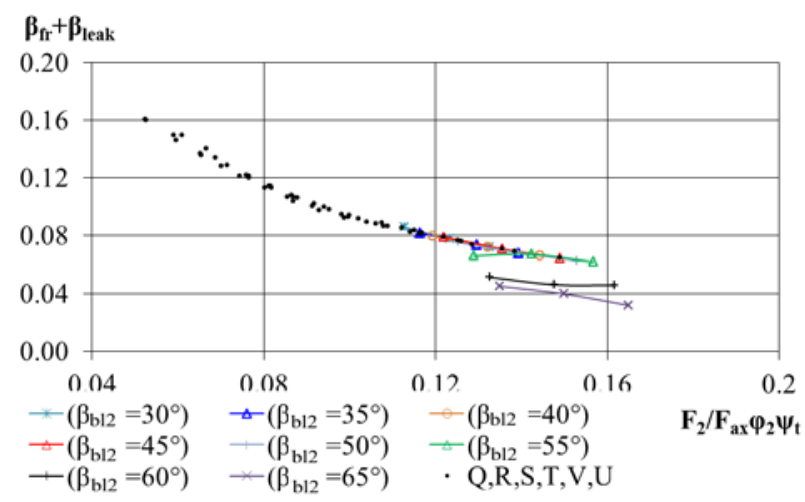

Fig. 10. Comparison of friction loss and leakage complex for stages Q, R, S, T, U, V and stage Q-482 with different blade outlet angle.

The average approximation error by the equation (7) is $1.3 \%$. Figure 10 shows that with an increase in the blades outlet angle, and accordingly the pressure of the impeller, there is a sharp decrease in the complex of friction and leakage loss. This effect limits the use of the dependence within the limits of the conditional flow coefficient $0.006<\Phi_{\mathrm{d}}<0.020$ and the theoretical pressure coefficient $0.60<\psi_{\mathrm{t}}<0.72$, which coincides with the range of the studied stages.

\section{Results}

The validation of generalizing dependencies was carried out on the basis of data not participating in their compilation. Figure 11 shows a good match between calculation and simulation. The average relative error in the generalizing dependence is $\delta\left(\psi_{\mathrm{t}}\right)=1.19 \%, \delta\left(\psi_{\mathrm{i}}\right)=$ $0.96 \%$. The average relative error in the generalizing dependence in comparison with experimental data is $\delta$ $\left(\psi_{\mathrm{i}}\right)=3.63 \%$.

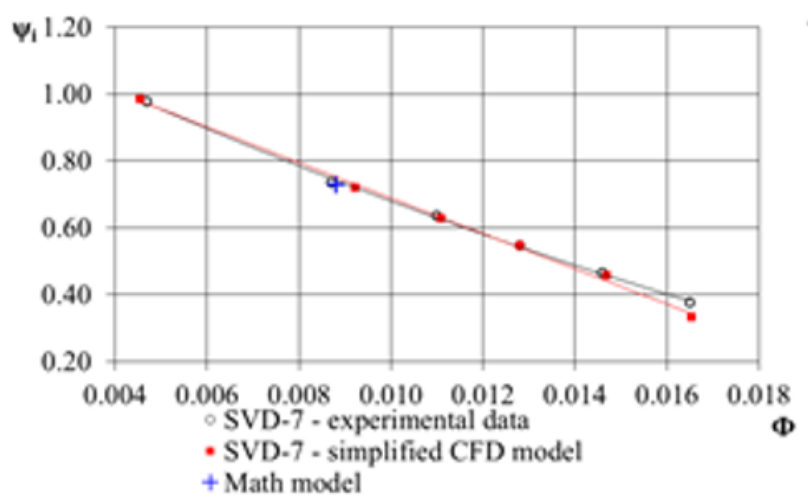

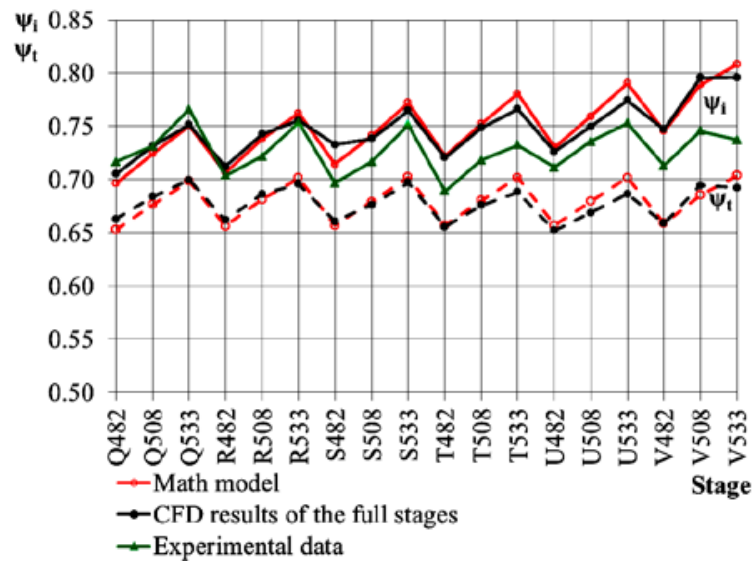

Fig. 11. Comparison of simulation results with the values obtained from the generalizing dependence and experimental data.

Since the simulation data for the detailed stages participated in the development of the equation for calculating the complex of friction and leakage loss, the data for the tested stages SVD-7 and SVD-8 CVRE (Compressor, vacuum and refrigeration engineering) research group [5] were used, not used in compiling a generalizing dependence of the theoretical pressure and the complex of friction and leakage loss.

Figure 12 compares the characteristics of the internal pressure coefficient with experimental data and the results of calculations by generalizing dependencies and modeling for stages SVD-7 and SVD-8.

The relative error for the low stage impeller in the calculated mode is $\delta\left(\psi_{\mathrm{i}}\right)=0.68 \%$ for SVD-7 and $\delta\left(\psi_{\mathrm{i}}\right)$ $=0.74 \%$ for SVD- 8 .

The use of generalizing dependences for the theoretical head coefficient and the set of friction and leakage loss for low-flow centrifugal compressors impellers showed good consistency on the independent experimental data, which indicates the reliability of the research results.

\section{Discussion}

A math model is developed in the form of generalizing dependences for calculating the theoretical head

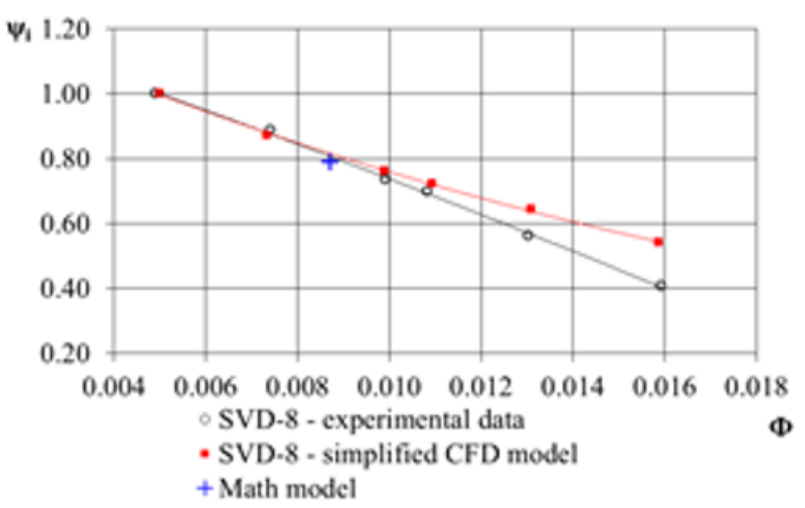

Fig. 12. Experimental characteristics of the internal pressure coefficient in comparison with the result of calculation by generalizing denendencies and modeling for the stages SVD-7 and SVD-8. 
coefficient $\psi_{\mathrm{t}}$ of low-flow impellers in the design mode for the centrifugal compressors stages of thermal power plants booster compressor stations. The application of the dependence in the range of the conditional flow coefficient is from $0.008 \div \Phi_{\mathrm{d}} \div 0.02$, the conditional Mach number is from $0.5 \div M_{u} \div 0.8$, the number of blades is from $12 \div 2 \div 20$, the blades outlet angle is from $30^{\circ} \div \beta_{\mathrm{bl} 2} \div 50^{\circ}$, the relative hub diameter is $0.3 \div \bar{D}_{h} \div 0.5$, the blade height ratio at the inlet and outlet is $0.5 \div b_{1} / b_{2} \div 1.0$. The feature of the proposed dependence is the generalization of the viscous three-dimensional flow modelling of validated simplified low-flow rate impellers numerical models results, taking into account viscous effects arising from the interaction of the flow core with the low-energy zone and secondary flows from bounding surfaces.

A generalizing relationship has been developed for calculating the complex of friction and leakage loss $\left(\beta_{\mathrm{fr}}\right.$ $\left.+\beta_{\text {leak }}\right)$ of low-flow rate impellers in the zone of optimal operation based on the consideration of viscous threedimensional flow in validated detailed numerical models of 18 low-flow stages. The application of the dependence in the range of the conditional flow coefficient is from $0.006<\Phi_{\mathrm{d}}<0.02$ and the theoretical head coefficient is $0.60<\psi_{\mathrm{t}}<0.72$.

The reliability of the calculation by generalizing dependencies is ensured by validation of the independent stages of the $Q, R, S, T, U, V$ and SVD-7, SVD-8 series. The arithmetic average relative error in calculating the theoretical pressure coefficient is not more than $2 \%$.

The results of the work were obtained using computational resources of Peter the Great SaintPetersburg Polytechnic University Supercomputing Center (www.spbstu.ru)

\section{References}

1. Seleznev, K. P. Centrifugal Compressors. L., Publishing house «Mashinostroenie»,- 271 p. (In Russ.) (1982).

2. Rice V.F. Centrifugal Compressor Machines / L., Publishing house «Mashinostroenie», 336 p. (In Russ.) (1964).

3. Galerkin Yu.B., Karpov A.N. Compressor Engineering and Pneumatics [Kompr. Tekh. Pnevm.], 6. p. 27. (In Russ.) (2011).

4. K. P. Seleznev and L. Y. Strizhak. Chemical and Petroleum Engineering. 31. № 11. pp. 585-595. (1995) https://doi.org/10.1007/BF01155742

5. Strizhak, L.Ya. / p.p. 233 - 286. Proceedings of the scientific school of compressor engineering SPbGPU under edition Y.B. Galerkin- 670 p. (In Russ.) (2010).

6. N. Sadovskiy, L. Strizhak, A. Simonov and M. Sokolov. MATEC Web Conf., 245 09004. (2018). https://doi.org/10.1051/matecconf/201824509004

7. V. M. Ivanov, Y. V. Kozhukhov, and A. M. Danilishin. AIP Conference Proceedings 2141, 030064 (2019); https://doi.org/10.1063/1.5122114
8. Paroubek, J., Cyrus, V., and Kync`1, J. ASME. J. Turbomach. 117(4): 585-592. (1995). https://doi.org/10.1115/1.2836572

9. K. P. Seleznev, L. Ya. Strizhak, E. S. Remezova, V. Riss, and D. Shmidt, Compressor Engineering and Pneumatics [Kompr. Tekh. Pnevm.], No. 3, 40-45 (1994).

10. I, Biba \& A, Nye \& Liu, Zheji. International Journal of Rotating Machinery. 8. (2002) 10.1080/1023620291910734.

11. Yablokov A.M., Sadovskiy N.I., Kozhukhov Y.V. Territorija "NEFTEGAS" [Oil and Gas Territory]. ;(5):28-35. (In Russ.) (2019)

12. A. Yablokov, I. Yanin, A. Danilishin and A. Zuev. MATEC Web Conf., $245 \quad$ (2018) 09002. https://doi.org/10.1051/matecconf/201824509002

13. Hazby, H., Casey, M., and Březina, L. ASME. J. Turbomach. 141(9): $091006 . \quad$ (2019); https://doi.org/10.1115/1.4043786

14. Kiryll Kabalyk, Władysław Kryłłowicz. Transactions IFFM 131(2016) 97-109

15. Lettieri, C., Baltadjiev, N., Casey, M., and Spakovszky, Z. ASME. J. Turbomach.; 136(8): 081008. (2014). https://doi.org/10.1115/1.4026322

16. Yongsheng Wang, Feng Lin, Chaoqun Nie, and Abraham Engeda, International Journal of Rotating Machinery, vol. 2013, Article ID 293486, 12 pages, (2013). https://doi.org/10.1155/2013/293486.

17. Wang, Z., Xi, G. \& Liu, Q. Sci. China Technol. Sci. 58: 29. (2015) https://doi.org/10.1007/s11431-0145697-8

18. Izmaylov, R., Lebedev, A. IOP Conference Series: Materials Science and Engineering, 90 (1), (2015) 012052. https://doi.org/10.1088/1757899X/90/1/012052

19. Lebedev, A., Gileva, L., Danilishin, A., Sokolov, M. MATEC Web of Conferences, 245, (2018) 09010. https://doi.org/10.1051/matecconf/201824509010

20. Galaev, S.A., Ris, V.V., Simoyu, L.L., Smirnov, E.M. Experience in numerical simulation of turbulent wet-steam flow in the last stage of a highpower condensing turbine under conditions defined by full-scale experiments at a power plant. 12th European Conference on Turbomachinery Fluid Dynamics and Thermodynamics, ETC 2017.

21. Ivanov, E.A., Zharkovsky, A.A., Borshchev, I.O., Svoboda, D.G. AIP Conference Proceedings 2141, 030063 (2019). https://doi.org/10.1063/1.5122113

22. Ivanov V.M., Kozhukhov Y.V. Danilishin A.M., Sadovskiy N.I. Novoe v rossijskoj e`lektroe nergetike [New in the Russian electric power industry], 6. C. 12-19, (In Russ.) (2019).

23. Wilcox, D.C. AIAA Journal, vol. 26, no. 11, pp. 1299-1310. (1988)

24. Galerkin Yu.B., Rextin F.S. Research methods of the centrifugal compressor machines M., Publishing house Mashinostroenie, - 304 p. (In Russ.) (1969). 